Journal of Mathematics and Statistics 3 (3): 80-82, 2007

ISSN 1549-3644

(C) 2007 Science Publications

\title{
Markov Property of the Solution of the Stochastic Generalized Equations
}

\author{
Khaldi Khaled \\ Department of Mathematics, Faculty of Sciences \\ Boumerdes University, 35000, Boumerdes, Algeria
}

\begin{abstract}
Some models of probabilities are described by generalized stochastic equations. These models (like that prediction) lead to the resolution of boundary problems for random distributions (generalized equations). We are interested in the equation $\mathrm{Lx}=\mathrm{f}$ in $\mathrm{S} \subset \mathrm{IR}^{\mathrm{d}}$ where $L$ is a linear operator, $f$ is a random distribution and to the class of boundary conditions on the frontier $\Gamma=\partial \mathrm{S}$ in order to define for the corresponding boundary conditions. The resolutions of boundary problems for random distributions lead to the Markov property for the solution of these equations.
\end{abstract}

Keywords: Linear operator, stochastic equation, stochastic distribution, Markov property

\section{INTRODUCTION}

The Markov property can for stochastic generalized equations be studied from several different angles, see, e.g. Levy (1956), Kallianpur and Mandrekar (1974), Rozanov (1981, 1987), Khaldi (1989, 2000). In this paper we consider the classical case where $\mathrm{L}$ is a linear operator.

The boundary problems for the linear equations of the type:

$$
\operatorname{Lx}(\mathrm{t})=\mathrm{f}(\mathrm{t}), \mathrm{t} \in \mathrm{S}
$$

where $L$ is a linear operator, lead to search the distribution $x(t), \mathrm{t} \in T$, in $\mathrm{T} \subseteq \mathrm{E}^{\mathrm{d}}$, containing the domain $T \subseteq E^{d}$, such as

$$
\mathrm{x}(\mathrm{t})=\mathrm{v}(\mathrm{t}), \mathrm{t} \in \mathrm{T} \backslash S
$$

where $T \backslash S$ is the additional of $S$ in $T$.

So that the problem admits a (only) solution, the distributions $f(t)$ and $v(t)$ as the solution $x(t)$ have to belong to a certain class which we shall describe more low. The solution $x(t)$ is bound to the operator $L$ verifying the equation:

$$
\mathrm{L} \xi(\mathrm{t})=\xi^{*}(\mathrm{t}), \mathrm{t} \in \mathrm{T}
$$

where $\xi(\mathrm{t}) \mathrm{t})$ and $\xi^{*}(\mathrm{t})$ are distributions with values in Hilbert's space $H$.

By distribution with values in $\mathrm{H}$, we understands a linear continuous application:

$\xi: \varphi \in \mathrm{C}_{0}^{\infty}(\mathrm{T}) \rightarrow(\varphi, \xi) \in \mathrm{H}$
In first, we are interested in the properties of the solution $\xi(\mathrm{t})$ of the boundary problem (3) that we prolong in $\mathrm{W}^{*}=\overline{\mathrm{C}_{0}^{\infty}(\mathrm{T})}$ space of distributions $\mathrm{u}=(\varphi, \mathrm{u}), \varphi \in \mathrm{C}_{0}^{\infty}(\mathrm{T})$. The distribution $\mathrm{u} \in \mathrm{C}_{0}^{\infty}(\mathrm{T})$ is, $(\varphi, \mathrm{u})=\int_{\mathrm{T}} \varphi(\mathrm{t}) \overline{\mathrm{u}(\mathrm{t})} \mathrm{dt}$

we define for a some set $S((\mathrm{~S} \subset \mathrm{T})$ the space

$$
\mathrm{H}_{+}(\mathrm{S})=\left\{(\mathrm{u}, \xi), \mathrm{u} \in \mathrm{W}^{*}, \operatorname{Supp} \mathrm{u} \subseteq \overline{\mathrm{S}}\right\}
$$

\section{MAIN RESULTS}

The solution $\xi(\mathrm{t})$ of the equation (3) for a local operator $L$ possesses the following Markov property: for everything $\mathrm{S} \subseteq \mathrm{T}$ with border $\Gamma=\partial \mathrm{S}$, the projection of the space $\mathrm{H}_{+}(T \mid S)$ in $\mathrm{H}_{+}(S)$ coincides with $\mathrm{H}_{+}(\Gamma)$.

One notices that an equation of the type (3) with $\mathrm{L}=1^{*} 1$ and $\xi^{*}(\mathrm{t})=1^{*} \eta(\mathrm{t})$ occupies an important place in the theory of the stochastic differential equations of the type $\mathrm{L} \xi(\mathrm{t})=\eta(\mathrm{t}), \mathrm{t} \in \mathrm{T}$, where $l$ is a differential linear operator, $\eta(\mathrm{t})$ a distribution in $H$ (Hilbert's space) called "white noise" in $\mathrm{t} \in \mathrm{T}$.

For every $\mathrm{u} \in \mathrm{C}_{0}^{\infty}(\mathrm{T})$, the distribution $\mathrm{Lu}=(\varphi, \mathrm{Lu}), \varphi \in \mathrm{C}_{0}^{\infty}(\mathrm{T})$ is a positive linear application $u \rightarrow L u:$ 
$(\varphi, L u) \geq 0$

with $\|\varphi\|=(\varphi, \mathrm{Lu})^{\frac{1}{2}}$ and one considers the space de Hilbert's $W$, completed of $\mathrm{C}_{0}^{\infty}(\mathrm{T})$ by the scalar product $\langle\mathrm{u}, \mathrm{v}\rangle=(\mathrm{u}, \mathrm{Lv}), \mathrm{u}, \mathrm{v} \in \mathrm{C}_{0}^{\infty}(\mathrm{T})$

By using (8), one corresponds to every $\mathrm{v} \in \mathrm{W}$ the distribution $L v$ :

$L v=(\varphi, \mathrm{Lv})=\langle\varphi, \mathrm{v}\rangle, \varphi \in \mathrm{C}_{0}^{\infty}(\mathrm{T})$

and one introduces $W^{*}$ (set of distributions $L v$

prolonged by continuance on $W$ ):

$L v=(u, L v)=\langle u, v\rangle$

It is evident that $\mathrm{W}^{*}$ is the dual of $W$ and exactly $\mathrm{W}^{*}$ is the set of functional linear on $\mathrm{W}$ described by (9), with $\|\mathrm{Lv}\|=(\mathrm{Lv}, \mathrm{Lvu})^{\frac{1}{2}}=\operatorname{Supp}_{\|\varphi\|=1}\langle\varphi, v\rangle$.

The equation (1) is understood in the sense $(\varphi, \mathrm{Lx})=\langle\varphi, \mathrm{f}\rangle, \varphi \in \mathrm{C}_{0}^{\infty}(\mathrm{S})$

and the boundary conditions (2) as $(\mathrm{u}, \mathrm{x})=(\mathrm{u}, \mathrm{v}), \mathrm{u} \in \mathrm{W}^{*}, \operatorname{Supp} \mathrm{u} \subseteq \mathrm{T} \backslash S$

For the search for the solution of the problem (1)(2), one considers the Hilbert's space $H$ defining the isometric application

$\xi^{*}: u \in W \rightarrow\left(u, \xi^{*}\right) \in H$

defined by (3).

The application (4) maybe identified with the isometric application

$\xi: u \in W^{*} \rightarrow(u, \xi)=\left(L^{-1} u, \xi^{*}\right) \in H$

We define $H(S)$ and $H^{*}(S)$ respectively by $H(S)=\overline{\left((\varphi, \xi), \varphi \in C_{0}^{\infty}(S)\right)}$ and $H^{*}(S)=\overline{\left(\left(\varphi, \xi^{*}\right), \varphi \in C_{0}^{\infty}(S)\right)}$.

It is evident that $H(T)=H^{*}(T)$. Afterward, one define $H=H(T)$.

That is to say $\{\xi, \eta\}$ the scalar product of $\xi$ and $\eta \in H$. One has so

$\left\{\left(\varphi, \xi^{*}\right),(u, \xi)\right\}=\left\{\left(\varphi, \xi^{*}\right),\left(L^{-1} u, \xi^{*}\right)\right\}$ $=\left\langle\varphi, L^{-1} u\right\rangle=\left(\varphi, L L^{-1} u\right)=(\varphi, u)$

The condition $(u, \xi) \perp\left(\varphi, \xi^{*}\right),\left(\varphi \in C_{0}^{\infty}(S)\right)$, for an opened set $S \subseteq T$ is equivalent in $\operatorname{Supp} u \subseteq T \backslash \mathrm{S}$. By indicated by $H^{*}(S)^{\perp}=H^{*}(T) \ominus H^{*}(S)$, one has
$\mathrm{H}_{+}(T \mid S)=H^{*}(S)^{\perp}$ where

$\mathrm{H}_{+}(T \backslash S)=\{(v, \xi), v \in W$, Supp $u \subseteq T \backslash \mathrm{S}\}$ because

$\left\{\left(v, \xi^{*}\right),(u, \xi)\right\}=(v, u)=\overline{(u, v)}$

that one deducts of (15) by passage on the limit of $\varphi \rightarrow v \in W$.

Theorem 1: The unique solution $x \in W$ of the problem (1)-(2) is given by:

$x=(u, x)=(g, f)+\left(\Pi_{\Gamma}^{+} x, v\right)$

Where $\Pi_{\Gamma}^{+}$is the operator of projection on $H_{+}(\Gamma)$ and $g$ the solution of the equation $L^{*} g=x-\Pi_{\Gamma}^{+} x$, Supp $u \subseteq \bar{S}$.

Proof: $\quad$ As $\quad H=H(S)^{\perp} \oplus H(\bar{S})$ $=H(\bar{S})^{\perp} \oplus H_{+}(\Gamma) \oplus H(T \backslash S)^{\perp}$, every $x \in H \quad$ can be written under the shape $x=x_{1}+x_{2}+x_{3}$ where $x_{1}, x_{2}$ and $x_{3}$ are the orthogonal projections of $x$ on the subspaces $H(\bar{S})^{\perp}, H_{+}(\Gamma)$ and $H(T \backslash S)^{\perp}$. One has then $(u, x)=\left(u, x_{1}\right)+\left(u, x_{2}\right)+\left(u, x_{3}\right), \quad x \in H(\bar{S})^{\perp} . \quad$ As $(u, x)=\left\langle L u,\left(L^{*}\right)^{-1} x\right\rangle=\left\langle L^{*} L u, x\right\rangle=\left\langle u,\left(L^{*} L\right)^{-1} x\right\rangle$, one has $\left(u, x_{3}\right)=\left\langle f,\left(L^{*}\right)^{-1} x_{3}\right\rangle \quad$ because $\quad \forall y \in L^{*} H(T \backslash S)^{\perp}$, $(u, y)=\langle L u, g\rangle, \quad g \in H(T \backslash S)^{\perp}, \quad g=\left(L^{*}\right)^{-1} y . \quad$ It remains to show that $\langle L u, g\rangle=\langle f, g\rangle, g \in H(T \backslash S)^{\perp}$. The equation $L x=f$ means $(\varphi, L x)=(\varphi, f)$, $\varphi \in C_{0}^{\infty}(S)$ or $L x-f \in H(T \backslash S)^{\perp}$. As a consequence $\langle L x-f, g\rangle=0, g \in H(T \backslash S)^{\perp}$ and so any solution of the equation $L x=f$ can be represented by $(u, x)=\left(u, x_{2}\right)+\left(f,\left(L^{*}\right)^{-1} x_{3}\right) \quad$ or $\quad(u, x)=\left(u, x_{2}\right)+\langle f, g\rangle \quad$ where $g=\left(L^{*}\right)^{-1}\left(x-x_{2}\right)$ with $x_{2}=\Pi_{\Gamma}^{+} x$.

Theorem 2: The equation

$L x(t)=0, t \in S$

where $L$ is a local operator local with the boundary conditions

$(u, x)=(u, v), u \in W^{*}, \operatorname{Supp} u \subseteq \Gamma$ 
possesses a unique solution $x \in W$ in $S$.

Proof: As one say that the solution is written by $x=(u, x)=(g, f)+\left(\Pi_{\Gamma}^{+} x, v\right)$. It remains to check that all solution $x \in W$ with null boundary conditions is egal to 0 in $S$. The condition (18) give $\left(x, \xi^{*}\right) \perp H^{*}\left(S_{1}\right), S_{1}=S$ and $0=(u, x)=\langle u, L x\rangle_{*}=\left\{(u, \xi),\left(x, \xi^{*}\right)\right\}$, Supp $u \subseteq \Gamma$ mean that $\left(x, \xi^{*}\right) \perp H_{+}(\Gamma)$.

The decomposition $H=H(\bar{S})^{\perp} \oplus H_{+}(\Gamma) \oplus H(T \backslash S)^{\perp}$ implies $\left(x, \xi^{*}\right) \in H^{*}(T \backslash S)=H_{+}(S)$. It means that $(u, x)=\left\{(u, \xi),\left(x, \xi^{*}\right)\right\}=0$, Supp $u \subseteq \bar{S}$.

Theorem 3: The equation (3) with a local operator $L$ possesses the Markov property.

Proof: $\quad$ As $\quad H_{+}(\Gamma)=H^{*}(S \cup(T \backslash \bar{S}))^{\perp} \quad$ where $H^{*}(S \cup(T \backslash \bar{S}))^{\perp}=H^{*}(S) \oplus H^{*}(T \backslash S)$ direct sum of the two sub-spaces $H^{*}(S)$ and $H^{*}(T \backslash S)$ because $\forall y \in C_{0}^{\infty}(S \cup(T \backslash \bar{S})), \quad \varphi=\varphi_{1}+\varphi_{2}, \quad \varphi_{1} \in C_{0}^{\infty}(S)$ and $\varphi_{2} \in C_{0}^{\infty}(T \backslash \bar{S})$, which, as elements of $W$ are orthogonal: $\quad\left\langle\varphi_{1}, \varphi_{2}\right\rangle=\left\langle\varphi_{1}, L \varphi_{2}\right\rangle=0$. One has consequently $\left\{\left(\varphi_{1}, \xi^{*}\right),\left(\varphi_{2}, \xi^{*}\right)\right\}=\left\langle\varphi_{1}, \varphi_{2}\right\rangle=0$. We have so the orthogonal decomposition $H=H(\bar{S})^{\perp} \oplus H_{+}(\Gamma) \oplus H(T \backslash S)^{\perp} \quad$ in $\quad$ which $H^{*}(S) \oplus H^{*}(\Gamma)=H^{*}(T \backslash S)^{\perp}=H_{+}(S) \quad$ and $H_{+}(\Gamma) \oplus H^{*}(T \backslash \overline{\mathrm{S}})=H^{*}(S)^{\perp}=H_{+}(T \backslash \overline{\mathrm{S}}) . \quad$ By indicating by $\Pi_{+}(S)$ and $\Pi_{+}(T \backslash \bar{S})$ the orthogonal projections on $H_{+}(S)$ and $H_{+}(T \backslash \overline{\mathrm{S}})$ respectively, one has $\Pi_{+}(S) H_{+}(T \backslash \overline{\mathrm{S}})=H_{+}(T \backslash \overline{\mathrm{S}}) \Pi_{+}(S)=H_{+}(\Gamma)$.

That means that the distribution $\xi(t)(\mathrm{t})$ possesses, in the space of Hilbert, the Markov property.

\section{REFERENCES}

1. Brzezniak, Z. and T. Zastawniak, 1999. Basic Stochastic Processes. Springer-Verlag.

2. Kallianpur, G. and U. Mandrekar, 1974. The Markov property for generalized Gaussian random fields. Ann. Inst. Fourier, 2: 143-167.

3. Khaldi, K., 1989. Boundary problems for local limited operators in Hilbert's spaces. Stochastic processes, mathematical statistics and applications. University of Moscow, pp: 31-33.

4. Khaldi, K., 2000. Boundary problems for stochastic equations and stochastic Markov fields. Proc. 3rd Intl. Conf. on Appl. Math. Engg. Sci., Casablanca.

5. Leveque, O., 2004. Cours de Probabilités et Calcul Stochastique. EPFL.

6. Levy, P., 1956. A special problem of Gaussian random functions. Proc. 3rd Berkeley Symp. Math. Stat. Prob., 2: 133-175.

7. Oksendal, B., 2000. Stochastic Differential Equations. Springer-Verlag.

8. Rozanov, Y., 1987. Some boundary problems for pour differential generalized equations. Math. Zametki, 1: 110-118.

9. Rozanov, Y., 1987. Boundary problems for stochastic partial differential equations. Universitat Bielfeld. Forschungszentrum Bielfeld Bochum Stochastic, No. 23.

10. Rozanov, Y., 1981. Stochastic Markov Fields. Naouka. Moscow. 